\title{
Hábitos higiénicos vulvo-vaginales de consultantes ambulatorias en gineco-obstetricia
}

\author{
Marcia López A. ${ }^{1}$, Felipe Ubilla $S .^{2}$, Cristián Morales $S .{ }^{2}$, Pedro Zitko $M .^{3}$, Viviana Burgos \\ M. ${ }^{4}$, Ma. Angélica Rivera A. ${ }^{4}$ \\ ${ }^{1}$ Departamento de Obstetricia y Ginecología, Facultad de Medicina Occidente, Universidad de Chile, Hospital San Juan \\ de Dios. ${ }^{2}$ Programa en Ginecología y Obstetricia, Facultad de Medicina Occidente, Universidad de Chile, Hospital San \\ Juan de Dios. ${ }^{3}$ Facultad de Medicina, Universidad Diego Portales, Unidad de Estudios Asistenciales, Complejo Asisten- \\ cial Barros Luco-Trudeau. ${ }^{4}$ Médicos Ginecología-Obstetricia, actividad privada.
}

\section{RESUMEN}

Objetivo: Determinar la frecuencia y tipos de hábitos higiénicos vulvo-vaginales en mujeres chilenas. Métodos: Estudio de corte transversal en 271 usuarias adultas que asistieron a centros ginecológicos primarios del sistema público y privado. Médico aplicó una encuesta de 7 preguntas con alternativas de respuestas cerradas para pesquisar la frecuencia y tipo de hábitos higiénicos íntimos. Se realizó estadística descriptiva utilizando modelos univariados y multivariados, expresando la magnitud de asociación como Odds Ratio. Se seleccionaron los modelos multivariados con mayor varianza mediante selección por pasos. Resultados: Un $25 \%$ lava sus genitales una vez/día, $32 \%$ dos veces/día, $28 \%$ tres veces/día y $13 \%$ cuatro o más veces/día. Las mujeres del sistema público tienden a un aseo genital con frecuencias de dos a tres veces al día, mientras que las del sistema privado mayormente una y dos veces al día. El 56\% de las mujeres, especialmente aquellas atendidas en consultas privadas, utiliza algún otro elemento adicional al agua, preferentemente jabones especiales. Las mujeres mayores y postmenopáusicas se asociaron a lavados genitales con elementos adicionales al agua. Las diabéticas presentaron una probabilidad 3 veces mayor de usar un jabón especial que las no diabéticas. Conclusiones: Mujeres chilenas de ambos sistemas de atención, realizan aseo íntimo con una frecuencia entre una y tres veces al día en el 85\% de las encuestadas. El $56 \%$ de ellas y el $75,6 \%$ de las que consultan en el sistema privado utilizan para el aseo genital algún otro elemento adicional al agua, preferentemente jabones especiales.

\section{PALABRAS CLAVE: Higiene íntima, hábitos higiénicos, autocuidado}

\section{SUMMARY}

Objective: To determine frequency and types of vulvovaginal hygienic habits that chilean women have. Methods: Cross-sectional study in 271 adult women who attended primary gynecological centers of public and private systems. A survey of 7 questions with closed-answer alternatives was undertaken by a physician in order to determine frequency and type of intimate hygiene. Descriptive statistics were performed by using univariate and multivariate models, expressing the magnitude of association as Odds Ratio. Multivariate models with higher variance were selected by stepwise selection. Results: $25 \%$ washed their genitals once a day, $32 \%$ twice a day, $28 \%$ three times a day and $13 \%$ four or more times a day. Women attending public centers tend to wash their genitals two or three times a day, while the ones attending private centers do this mostly once or twice a day. $56 \%$ of women - especially those treated in private centers - use additional elements apart from water, preferably special soaps. Older and postmenopausal women were associated with the use of additional elements other than water. Diabetics were 3 times more likely to use a special soap than non-diabetics. Conclusions: $85 \%$ of chilean women of both health-care systems perform intimate 
hygiene between one and three times a day; $56 \%$ of them and $75.6 \%$ of those attending private centers use elements other than water, preferably special soaps.

\section{KEY WORDS: Intimate hygiene, hygienic habits, self care}

\section{INTRODUCCIÓN}

La higiene íntima de la mujer forma parte de los procesos de autocuidado de la salud, y su aprendizaje se ha realizado históricamente por la enseñanza que imparten las madres a sus hijas. Dentro de sus objetivos están la prevención de infecciones, brindar comodidad y bienestar, además de mejorar la calidad de vida de la mujer.

La cercanía del tracto urogenital inferior de la mujer a la región anal lo expone al contacto con microorganismos provenientes del intestino, lo cual favorece la aparición de infecciones vulvo-vaginales y del tracto urinario. Asimismo, la actividad sexual expone esta área a microorganismos provenientes del tracto genitourinario masculino y al riesgo de infecciones de transmisión sexual.

El autocuidado tiene que ver con el conjunto de decisiones y prácticas adoptadas por el individuo para ejercer un mayor control sobre su bienestar físi$\mathrm{co}$, psicológico y espiritual (1). Se trata de un proceso voluntario, que le permite a la persona convertirse en sujeto de sus propias acciones.

Los hábitos de higiene femeninos incluyen: baño y secado corporal diario, limpieza del área genital después de orinar o defecar, uso de elementos de protección menstrual, aseo genital en los días de menstruación e higiene relacionada con la actividad sexual.

Una correcta higiene íntima puede mantener o restablecer el pH vaginal normal y, por lo tanto, garantizar un ecosistema vaginal saludable libre de patógenos. Sin perjuicio de esto, debe considerarse que un lavado genital muy frecuente (más de 2 a 3 veces al día) y/o el uso de detergentes agresivos o limpiadores con un $\mathrm{pH}$ inadecuado (neutro o alcalino) remueven la capa de sebo que es esencial para proteger la superficie vulvo-vaginal que está expuesta a procesos inflamatorios causados por ataques físicos (microtraumas), químicos (jabones, alérgenos) e infecciosos (bacterias, hongos, virus y parásitos). Por estas razones, una protección íntima adecuada juega un rol clave en la mantención de una buena salud, y debe ser confiado a un detergente íntimo específico. Tales productos deben ser seleccionados tomando en cuenta la edad, estilo de vida y estado fisiopatológico de la mujer, ya que ellos pueden afectar el balance del ecosistema vaginal (2).

Hasta el momento son escasos los estudios sobre hábitos higiénicos en mujeres latinoamericanas y ninguno en Chile, y sus datos podrían aportar información útil para definir estrategias educativas encaminadas a mejorar el conocimiento en dichas mujeres, así como de los médicos que se ven enfrentados a sus interrogantes en la práctica profesional.

El objetivo de nuestro estudio fue determinar la frecuencia y tipo de hábitos higiénicos vulvo-vaginales de consultantes ambulatorias de la especialidad de ginecobstetricia que acudieron a centros de atención primaria del sistema público y privado en diferentes localizaciones de la ciudad de Santiago, Chile.

\section{SUJETOS Y MÉTODO}

Se desarrolló un estudio de corte transversal en usuarias de centros de atención primaria del sistema público y usuarias de consultas privadas de la especialidad de ginecobstetricia, en diferentes localizaciones de la ciudad de Santiago. A cada participante se le aplicó un instrumento para pesquisar la frecuencia y tipo de hábitos higiénicos vulvo-vaginales. El estudio fue aprobado por el Comité de Ética para investigación de Seres Humanos, de la Facultad de Medicina de la Universidad de Chile.

Muestra. La población objetivo correspondió a mujeres mayores de 18 años consultantes ambulatorias de la especialidad de gineco-obstetricia. El marco muestral se restringió por conveniencia a tres consultorios de atención primaria de salud ubicados en la comuna de Lo Prado donde se realiza atención de especialidad gineco-obstétrica, y a tres centros de atención ambulatoria privados ubicados en las comunas de Quilicura, Providencia y Las Condes, todos en la Región Metropolitana del país. La muestra fue tomada de la consulta espontánea de manera correlativa a partir de la fecha de inicio del período de recolección de datos de la investigación, hasta alcanzar el tamaño muestral deseado. El cálculo de tamaño muestral se realizó bajo el supuesto de la estimación de una prevalencia con varianza máxima (prevalencia 50,0\%), significación de 95\% en los intervalos de confianza y un error máximo de $10 \%$, resultando en 100 observaciones. Nuestro objetivo de tamaño muestral fue fijado en obtener al menos 200 observaciones, de las cuales se intencionó que al menos dos tercios correspondieran a consultas públicas y las restantes de los centros privados.

El único criterio de inclusión utilizado fue la edad de la participante mayor a 18 años. No se consideraron criterios de exclusión.

Recolección de datos. La recolección de datos se realizó mediante una encuesta. En una revisión de literatura hecha por los autores no se encontraron instrumentos estandarizados para medir frecuencia 
y tipo de hábitos higiénicos vulvo-vaginales, motivo por el cual se elaboró un instrumento propio. Este instrumento fue evaluado y modificado parcialmente por un médico con formación en epidemiología y con experiencia en la construcción de encuestas de salud. El instrumento resultante fue probado en 10 consultantes evaluando su comprensión y aplicabilidad. Además de la medición de frecuencia y tipo de hábitos higiénicos vulvo-vaginales, el instrumento consideró la evaluación del antecedente de menopausia y la presencia de diabetes mellitus, utilizando encabezados similares a los usados en la Encuesta Nacional de Salud 2009/2010 (MINSAL 2010) (3).

El instrumento final contó con 7 preguntas con alternativas de respuestas cerradas. La aplicación de cada encuesta tomó cerca de 2 minutos. La frecuencia de lavado genital se consultó preguntando "Pensando en un día normal, ¿con que frecuencia se lava usted los genitales?", con dos alternativas de respuestas: "a) Menos de una vez al día” y "b) Al menos una vez al día". A las mujeres que contestaron "Al menos una vez al día”, se les preguntó “¿Cuántas veces al día se lava los genitales normalmente?".

Los elementos utilizados en el lavado genital fueron consultados de la siguiente forma: "Cuando usted se lava los genitales, ..... a) Se los lava solo con agua, .....b) $O$ utiliza algún otro producto además del agua". A quienes contentaron la alternativa "b", se les pidió señalar el o los productos utilizados: "jabón común", "shampoo común", "gel común", "desodorante o perfume", "otro".

Los antecedentes de menopausia y diabetes mellitus fueron registrados como auto reporte: " $¿ U s-$ ted se encuentra en el período de menopausia, es decir le dejó de llegar la regla?”, y “¿Alguna vez un médico o profesional de la salud le diagnosticó diabetes o azúcar elevada en la sangre, y le dejaron tratamiento con remedios o fármacos?", respectivamente. También se consultó la fecha de nacimiento de la usuaria, además de registrar el lugar donde se realizó la entrevista: centro de atención público o privado.

La aplicación del instrumento se realizó previa invitación a participar y firma de consentimiento informado. La invitación y entrevista fue hecha por el médico que realizó la atención de especialidad una vez finalizada la consulta. En total participaron 5 médicos encuestadores. Quienes aplicaron la encuesta recibieron una inducción a la función, por parte de uno de los autores la cual tomó un tiempo cercano a 2 horas. Los datos recolectados por medio de la encuesta fueron digitados en planilla electrónica.

Análisis estadístico. Se realizó estadística descriptiva de cada una de las variables del estudio. Se determinó la prevalencia de cada frecuencia de lavados genitales colapsando las categorías con menores números. También se reporta la prevalencia de aseo "solo con agua", "usa otros elementos", y "usa jabón especial". Las prevalencias son presentadas como totales y estratificadas por sistema público y sistema privado.

Los factores asociados al uso de elementos adicionales al agua en el lavado genital, o los factores asociados al uso específico de jabones especiales, fueron explorados mediante modelos de regresión logística. En los modelos se incluyeron como factores asociados: la frecuencia de lavado (en 5 ó 4 estratos), edad (expresada en décadas), auto reporte de diabetes, auto reporte de menopausia, y sistema de atención (público - privado). Se realizaron modelos univariados y multivariados, expresando la magnitud de la asociación como Odds Ratio (OR). Adicionalmente, se exploraron términos de interacción buscando una magnitud de asociación diferente entre lavado y la frecuencia, edad, auto reporte de diabetes y auto reporte de menopausia según sistema de atención de la participante.

Además de los modelos señalados se seleccionaron los modelos multivariados con mayor varianza explicada, utilizando como procedimiento una selección por pasos (stepwise). Todas las estimaciones son reportadas con sus respectivos intervalos de confianza al 95\%. Las estimaciones fueron realizadas usando el software estadístico R 2.15.0.

\section{RESULTADOS}

Se entrevistó a 271 usuarias. Dado que en algunas encuestas se omitieron las respuestas a algunas preguntas se eliminaron 24 , siendo analizadas un total de 247 participantes válidos. La variable con mayor cantidad de datos perdidos correspondió a la frecuencia de aseo $(8,5 \%)$, diabetes $(1,1 \%)$, menopausia $(0,7 \%)$ y lavado $(0,7 \%)$.

De las participantes, $90(36,4 \%)$ pertenecieron al sistema privado. La media de edad fue 42,1 $\pm 14,8$ años, $15,4 \%$ declaró la presencia de diabetes, y un $39,7 \%$ en menopausia (Tabla I).

\section{Tabla I}

CARACTERÍSTICAS DE LA MUESTRA

\begin{tabular}{lcc}
\hline Variable & $\mathrm{n}$ & $\%$ \\
\hline Edad: $18-20$ años & 19 & 7,7 \\
Edad: $21-40$ años & 101 & 40,9 \\
Edad: $41-55$ años & 89 & 36,0 \\
Edad: $>$ 55 años & 38 & 15,4 \\
Diabetes & 38 & 15,4 \\
Menopausia & 98 & 39,7 \\
Sistema Privado & 90 & 36,4 \\
\hline
\end{tabular}

$\mathrm{n}=247$ mujeres 
En la Tabla II se presenta la frecuencia de lavado y de uso de otros elementos adicionales al agua. Se constata que cerca de un cuarto de la muestra declara lavarse una vez al día, un tercio dos veces al día, y cerca de otro cuarto tres veces al día. Un poco más de la mitad de las mujeres utiliza algún otro elemento adicional al agua, donde los jabones especiales acumulan la mayor frecuencia. Otros elementos utilizados corresponden a cloro $(2 \%)$, glicerina $(1,2 \%)$, y bálsamo, cloro/vinagre, jabón afrecho, jabón Popeye, polvos talco y vinagre ( $0,4 \%$ cada uno de ellos).

La distribución de la frecuencia de lavado presenta diferencias significativas al comparar la muestra entre mujeres que se atienden en el sistema público versus privado (Tabla II). Las primeras tienden a concentrarse en frecuencias de lavados de entre dos y tres veces al día, mientras que las usuarias del sistema privado se concentran en una y dos veces al día. El uso de otros elementos adicionales al agua, al igual que jabones especiales es más frecuente en mujeres atendidas en consultas privadas que públicas.

La frecuencia del lavado no evidenció asociación con el uso de elementos adicionales al agua (Tabla III). Al observar los OR de los modelos univariados y multivariados, se constata un comportamiento aparentemente no lineal, donde la mayores tendencias de uso de elementos adicionales al agua se observan en los estratos de frecuencias de lavado de una vez al día y cuatro o más veces al día. Mayor edad y menopausia se asociaron univariadamente al lavado con elementos adicionales al agua, aunque pierden significación estadística al incluirlas conjuntamente en el modelo multivariado. La presencia de diabetes no evidenció asociación con el lavado con elementos adicionales al agua. Por otro lado, las usuarias de atención privada presentaron una probabilidad mayor más de 3 veces que las usuarias del sistema público de usar un jabón especial. En el modelo de selección stepwise, se mantuvieron significativas tan solo las variables de menopausia y sistema de atención.

Respecto a la asociación entre el uso de jabones especiales y las otras variables mencionadas, ninguna de ellas - con excepción del sistema de atención - evidenció significación estadística, ya sea en el modelo univariado o multivariado (Tabla IV). Sin embargo, en la selección stepwise, las usuarias con auto reporte de diabetes presentaron un OR de cerca de 3 veces mayor probabilidad de uso de jabón especial que las mujeres sin auto reporte de diabetes. El sistema de atención privado mostró una clara asociación con el uso de jabones especiales en todos los modelos estudiados.

Ninguna de las interacciones exploradas entre sistema de atención y las demás covariables presentó significación estadística, ni para los modelos de uso de otros elementos adicionales al agua, ni para los modelos de uso de jabones especiales (Tablas $\vee$ y $\mathrm{VI}$ ).

\section{DISCUSIÓN}

La higiene íntima femenina constituye un elemento fundamental del autocuidado de la salud de la mujer, sin embargo la frecuencia con que se realiza y tipos de limpiadores utilizados además del agua pueden diferir en la población femenina según los hábitos higiénicos adquiridos desde la infancia, fundamentalmente a través de la madre, y los estilos de vida que lleva la mujer de hoy, lo cual incluye los hábitos relacionados con la actividad sexual.

En este estudio se constata, respecto de la frecuencia y tipos de hábitos higiénicos vulvo-vaginales, que cerca de un $25 \%$ de ellas declara lavar sus genitales una vez al día, un $32 \%$ dos veces al día, un $28 \%$ tres veces al día y un $13 \%$ cuatro o más veces al día. Las mujeres atendidas en el sistema público tienden a un aseo genital con frecuencias de dos a tres veces al día, mientras que las usuarias del sistema privado se concentran en una y dos veces al día. También se comprueba que el 56\% de las mujeres, especialmente aquellas atendidas en consultas privadas, utiliza algún otro elemento adicional al agua $(75,6 \%)$, donde los jabones especiales acumulan la mayor frecuencia. Además, las mujeres de mayor edad y postmenopáusicas se asociaron con lavados genitales con elementos adicionales al agua y las mujeres con diabetes mellitus presentaron una probabilidad 3 veces mayor de usar un jabón especial que las mujeres sin diabetes.

En un estudio de Cuevas y colaboradores (4), cuyo objetivo fue describir los hábitos higiénicos íntimos de 1.000 mujeres de 10 países latinoamericanos durante 2008 , se evidenció que el $95,4 \%$ de las mujeres encuestadas refirió realizar aseo corporal una o más veces al día; que el $71 \%$ evidenció una técnica adecuada de aseo perineal (de vulva a ano) y que el $90 \%$ usó el jabón barra para el aseo del área genital, siendo esa presentación la más utilizada, respecto de la líquida. Además, entre las mujeres encuestadas el $59 \%$ utiliza el mismo jabón tanto para el cuerpo como para el área genital y el $31 \%$ refirieron utilizar un jabón exclusivamente diseñado para el área genital. Por otro lado, el 83\% de las mujeres encuestadas limpia su área genital en relación al coito; el $16 \%$ antes de tener relaciones sexuales, el $40 \%$ después de tener actividad sexual y el $27 \%$ antes y después. Respecto del uso de elementos de aseo genital durante las relaciones sexuales, el $30 \%$ de las mujeres encuestadas refirió utilizar duchas vaginales. 


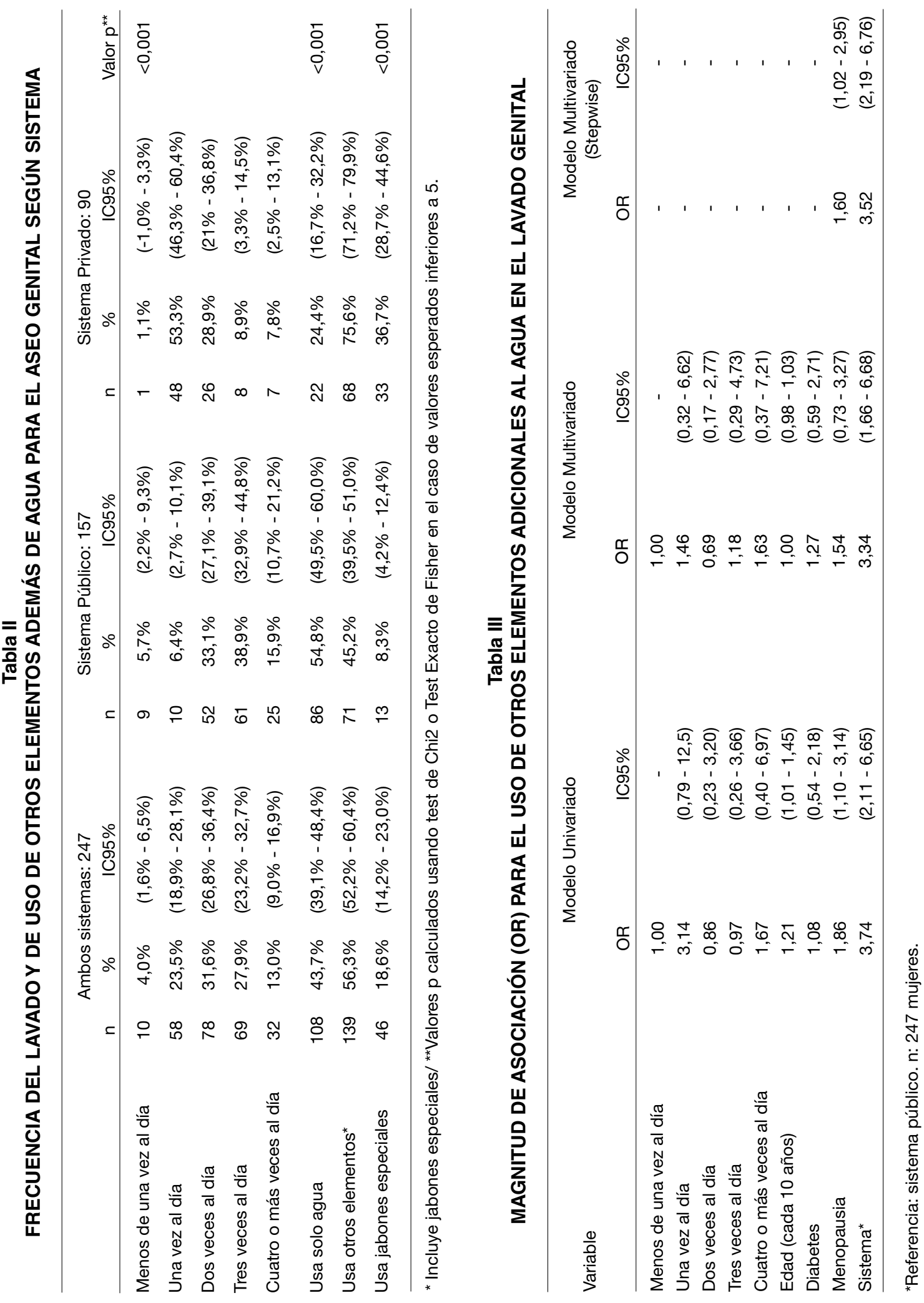




\section{Tabla IV}

MAGNITUD DE ASOCIACIÓN (OR) PARA EL USO DE JABÓN ESPECIAL EN EL LAVADO GENITAL

\begin{tabular}{lcccccc}
\hline Variable & \multicolumn{3}{c}{ Modelo Univariado } & \multicolumn{2}{c}{ Modelo Multivariado } & \multicolumn{2}{c}{$\begin{array}{c}\text { Modelo Multivariado } \\
\text { (Stepwise) }\end{array}$} \\
& OR & IC95\% & OR & IC95\% & OR & IC95\% \\
\hline Una o menos de una vez al día & 1,00 & - & 1,00 & - & - & - \\
Dos veces al día & 0,41 & $(0,18-0,91)$ & 0,76 & $(0,31-1,85)$ & - & - \\
Tres veces al día & 0,10 & $(0,03-0,36)$ & 0,29 & $(0,07-1,19)$ & - & - \\
Cuatro o más veces al día & 0,41 & $(0,14-1,23)$ & 1,06 & $(0,30-3,73)$ & - & - \\
Edad (cada 10 años) & 1,09 & $(0,88-1,3)$ & 1,00 & $(0,98-1,03)$ & - & - \\
Diabetes & 1,42 & $(0,60-3,3)$ & 2,56 & $(0,91-7,23)$ & 2,81 & $(1,06-7,44)$ \\
Menopausia & 1,09 & $(0,55-2,16)$ & 0,57 & $(0,22-1,48)$ & - & - \\
Sistema* & 10,8 & $(4,70-24,7)$ & 10,3 & $(3,99-26,7)$ & 11,4 & $(5,10-25,5)$ \\
\hline
\end{tabular}

${ }^{*}$ Referencia: sistema público. n: 247 mujeres.

\section{Tabla V \\ VALOR P PARA INTERACCIÓN ENTRE FRECUENCIA, EDAD, DIABETES Y MENOPAUSIA CON SISTEMA DE ATENCIÓN (PÚBLICO O PRIVADO)}

\begin{tabular}{lc}
\hline Variable & Valor $\mathrm{p}^{*}$ \\
\hline Menos de una vez al día & 0,855 \\
Una vez al día & 0,994 \\
Dos veces al día & 0,994 \\
Tres veces al día & 0,994 \\
Cuatro o más veces al día & 0,990 \\
Edad (cada 10 años) & 0,216 \\
Diabetes & 0,984 \\
Menopausia & 0,970
\end{tabular}

*No significativo. $\mathrm{n}: 247$ mujeres.

Tabla VI

VALOR P PARA INTERACCIÓN ENTRE FRECUENCIA, EDAD, DIABETES Y MENOPAUSIA CON SISTEMA DE ATENCIÓN (PÚBLICO O PRIVADO), PARA LAVADO CON JABÓN ESPECIAL

\begin{tabular}{lc}
\hline Variable & Valor $\mathrm{p}^{*}$ \\
\hline Una o menos de una vez al día & 0,723 \\
Dos veces al día & 0,503 \\
Tres veces al día & 0,588 \\
Cuatro o más veces al día & 0,809 \\
Edad (cada 10 años) & 0,069 \\
Diabetes & 0,169 \\
Menopausia & 0,511 \\
\hline
\end{tabular}

${ }^{\star}$ No significativo. $\mathrm{n}: 247$ mujeres.
El estudio SOPHY (5), realizado en Italia, evaluó el efecto de la higiene íntima sobre el $\mathrm{pH}$ vaginal, candidiasis, vaginosis bacteriana y actividad sexual en 2.641 mujeres, aleatorizadas en 6 subgrupos (pre-púberes, fértiles, embarazadas, periodo de lactancia, premenopausia y posmenopausia), permitió establecer que una higiene íntima correcta basada en un aseo genital con una frecuencia no mayor a 2-3 veces al día y la utilización de limpiadores naturales, no agresivos y de $\mathrm{pH}$ ácido, contribuyen a mantener y/o restaurar los valores fisiológicos del $\mathrm{pH}$ vaginal, evitando hacer desaparecer la capa de sebo protectora y el desbalance del ecosistema vaginal que permita el desarrollo de gérmenes y la aparición de patologías vulvo-vaginales de importancia clínica como las candidiasis y vaginosis bacterianas.

El estudio Epheso (6), el primer estudio epidemiológico sobre el $\mathrm{pH}$ vaginal de la población femenina española, publicado el 2008, constató que mantener el nivel de $\mathrm{pH}$ vaginal adecuado en cada periodo de la vida de la mujer está directamente relacionado con el bienestar, la satisfacción sexual y la ausencia de patologías. Así mismo el estudio demuestra que las alteraciones de $\mathrm{pH}$ son indicativas de diferentes problemas o infecciones vaginales, como por ejemplo la vaginitis. En este sentido, el estudio destaca que las mujeres que manifiestan una mayor satisfacción e interés sexual presentan valores de $\mathrm{pH}$ más bajos y equilibrados.

En el marco del tercer foro latinoamericano sobre higiene íntima femenina (7), realizado el 2009, se presentó un estudio de corte epidemiológico cuyo objetivo fue describir los hábitos higiénicos íntimos femeninos de un grupo de mujeres que acuden a la consulta externa ginecológica y determinar si estas prácticas de higiene pueden ser consideradas un factor de riesgo para el desarrollo de vaginosis bacteriana. De acuerdo con los resultados del 
estudio en que se evaluaron 403 casos y 602 controles, la aplicación de una técnica de aseo adecuada, en dirección de adelante hacia atrás, se asocia con una reducción del riesgo de vaginitis del $41 \%$. Además el $73 \%$ de las mujeres refiere utilizar siempre jabón en el aseo del área genital y en este sentido el uso de jabón líquido, exclusivo del área genital reducen el riesgo de vaginitis bacteriana.

Con el propósito de prevenir vaginosis de origen infeccioso las principales recomendaciones planteadas por los especialistas asistentes al tercer foro latinoamericano sobre higiene íntima femenina son (7): adoptar hábitos higiénicos adecuados que incluyen una correcta técnica de aseo con una frecuencia no mayor a 2-3 veces por día y con un limpiador no irritante que sea capaz de mantener o restablecer el $\mathrm{pH}$ vaginal ácido.

Nuestro estudio, hasta donde llega nuestro conocimiento, es el primero sobre hábitos higiénicos íntimos en mujeres chilenas que consultan habitualmente la especialidad gineco-obstétrica, tanto en el sistema público como privado. Sus datos podrían aportar valiosa información para definir estrategias educativas que permitan corregir comportamientos errados que pueden favorecer la aparición de infecciones vulvo-vaginales y por tanto la necesidad de utilizar fármacos antifúngicos y antibacterianos, contribuyendo al aumento del riesgo de resistencia. El hecho que el estudio se haya efectuado en pacientes bajo control sanitario regular, hace suponer que la educación sanitaria entregada por el especialista (particularmente en el sector privado) es insuficiente. También es posible presumir que el problema descrito es aún mayor en el resto de la población que no se controla usualmente con un especialista.

Una limitación de este estudio es la falta de cuantificación del impacto de los hábitos higiénicos observados sobre las patologías infecciosas vulvovaginales.

\section{CONCLUSIONES}

Mujeres chilenas que se atienden en consultas ginecológicas, tanto del sistema público como privado, realizan un aseo íntimo con una frecuencia entre una y tres veces al día en el $85 \%$ de las encuestadas. Se constata además que el $56 \%$ de ellas y en particular el $75,6 \%$ de las que consultan en el sistema privado utilizan para el aseo genital algún otro elemento adicional al agua, donde los jabones especiales acumulan la mayor frecuencia.

\section{REFERENCIAS}

1. Vivo positivo: Coordinadora Nacional de Agrupaciones y Organizaciones de Personas Viviendo con VIH/sida. Santiago, Chile. Disponible en: http://www. vivopositivo.org/portal/sitio/031.htm. Acceso: Abril de 2015.

2. Guashino S, Benvenuti C; SOPHY Study Group. SO$\mathrm{PHY}$ project: an observational study of vaginal $\mathrm{pH}$ and lifestyle in women of different ages and in different physiopathological conditions. Part I. Minerva Ginecol 2008;60:105-14.

3. MINSAL (2010). Encuesta Nacional de Salud. ENS Chile 2009-2010. Ministerio de Salud de Chile.

4. Cuevas A, Célis $C$, Hernández I, Herrán S, Paradas A, Paredes O, et al (Grupo MASIM). Resultados de una encuesta epidemiológica de hábitos de higiene íntima en mujeres latinoamericanas. Rev Obstet Ginecol Venez 2011;7(1):21-7.

5. Guashino S, Benvenuti C; SOPHY Study Group. SOPHY project: an observational study of vaginal $\mathrm{pH}$, lifestyle and correct intimate hygiene in women of different ages and in different physiopathological conditions. Part II. Minerva Ginecol 2008;6085):353-62.

6. Estudio EPHESO. Disponible en: http://www.jano.es/ jano/actualidad/ultimas/noticias/ejercicio/relaciones/ sexuales/ph/vaginal. Octubre 2008. Acceso: Abril de 2015.

7. Congress Report Symposia. 3er Foro Latinoamericano sobre Higiene Íntima Femenina. 18 al 20 de febrero de 2009. Varadero, Cuba. Higiene Íntima. Una necesidad real de la mujer latinoamericana. Parte 2, pp 2-7. Disponible en: http://www.lactacyd.com.co/lal/ pa/sp/layout.jsp?cnt=14A9C999-D676-4165-878A71A7FAA6AFE2. Acceso: Abril de 2015. 